\title{
Genetic Variability and Correlation Study for Yield and Quality Characters in Rice
}

\author{
Debashis Saren $^{1 *}$, Subhra Mukherjee ${ }^{1}$ and Rajib Tudu ${ }^{2}$
}

${ }^{I}$ Department of Genetics and Plant Breeding, BCKV, Mohanpur, Nadia, West Bengal, India

${ }^{2}$ Department of Entomology, BCKV, Mohanpur, Nadia, West Bengal, India

*Corresponding author

\section{Keywords}

Rice, Yield, Quality, GCV and PCV

\section{Article Info}

Accepted:

17 November 2019 Available Online: 10 December 2019

\section{A B S T R A C T}

Eleven rice genotypes were evaluated for different yield and quality parameters. On evaluation of parameters eight of them like Annada, IET 826, MTU 1010, Vandana, NC 1281, ND 97, IR 8 and PNR 381 were chosen as females (Line) and three parents like Satabdi, Pusa sugandha and IR 69705 were considered as males (tester). These lines and testers were combined following $\mathrm{L} \times \mathrm{T}$ crossing design to Estimates of heritability, Genetic Advance, Genetic Advance in percent were evaluated in this study. Yield plant $^{-1}$ showed significant positive correlation with number of effective tillers plant ${ }^{-1}$, panicle length, grains panicle ${ }^{-1}$, grains plant ${ }^{-1}, 1000$ grains weight, high to very high heritability was observed in most of the characters in parents and hybrids.

\section{Introduction}

Rice (Oryza sativa L.) is the most important cereal crop which is cultivated in the world, above fifty percent people used it as main food and its play important role as a staple food in Asia and Mediterranean Europe. Global coverage of rice cultivated area is over 159 million hectares producing 659.9 million tons per annum (FAO, 2007). More yield to provide sufficient food to poor people with assured protection from malnutrition future researches must be serious to make improvement for grain quality considering the demand coming from markets and consumers. Simultaneously plant breeders have to accept challenge to upgrade qualitatively of high yielding varieties to make them most remunerative.

The main yield and quality traits considered in the present investigation. Gelatinization temperature and amylose content are the two most important chemical quality features of rice which determine the texture and firmness of cooked rice. Gelatinization also determines 
the time taken to cook rice and affects water uptake, volume expansion and linear kernel elongation (Tomar and Nanda, 1982).

Majority of rice eaters prefer rice with intermediate amylose content and gelatinization temperature. Grain size, shape and appearance are important physical quality parameters that determine market value of rice. Long slender to extra long slender type is most preferred one, especially under basmati category which fetches highest price.

\section{Materials and Methods}

The field and laboratory experiments were conducted at the Jaguli instructional farm and the lab worked done at the department of Plant Breeding, Bidhan Chandra Krishi Viswavidyalaya, Mohanpur, Nadia, West Bengal, India. In the present study the materials comprises 8 parents viz., Annada, MTU 1010, Vandana, NC1281, ND 97, IR 8 and PNR 381 used as females (Line) and 3 Parents viz., Satabdi, Pusa sugandha and IR 69705 used as males (Tester) following the Line $\times$ Tester mating system (Kempthrone, 1957). The experiment was laid out in Randomized Block Design with three replications. The plant to plant distance were $25 \mathrm{~cm}$ and row to row were $30 \mathrm{~cm}$. The data were recorded on 10 randomly selected plants. Eleven genotypes were evaluated for yield and quality parameters. The different yield and quality parameters were plant height, number of effective tillers ${ }^{-1}$, panicle length $(\mathrm{cm})$, grains panicle $^{-1}$, grains plant $^{-1}, 1000$ grains weight (g), kernel length $(\mathrm{mm})$, kernel breadth $(\mathrm{mm})$, kernel length : breadth ratio, cooked kernel length $(\mathrm{mm})$, cooked kernel breadth $(\mathrm{mm})$, cooked kernel length : breadth ratio, Days to $50 \%$ flowering, days to maturity, yield plant ${ }^{-1}$, amylose content $(\%)$, gelatinization temperature, poor grain density (\%), average grain density (\%), above average grain density $(\%)$ and high grain density (\%).

\section{Results and Discussion}

The variability indeed is an outcome of genetic constitution of individuals within a population in relation to prevailing environment. Genetic variability may be evaluated on the basis of estimated values of parameters like genotypic variance, phenotypic variance, GCV, PCV, heritability, genetic advance etc. Data on these parameters for twenty one characters in parents and hybrids were represented in table 1 and 2 . High genotypic variances was observed for the characters grain plant ${ }^{-1}$ followed by plant height, days to maturity and high density grain both in parents and hybrids, though in hybrids genetic variance for all the characters were found to be comparatively less than that of parents. Marginal variations were evident between PCV and GCV for almost all the characters in parents and hybrids except gelatinization temperature in parents and all grades of rice grain for poor to high density. It can be suggested that all these characters were least influenced by environment except those characters which showed higher difference between PCV and GCV and phenotypic selection may provide desirable genetic improvement on these characters. The lower magnitude of genetic variation in hybrids as compared to total variation present in parents may be due to a few selected parents were considered to develop the hybrids. High to vary high heritability was observed in most of the characters in parents and hybrids except a few like kernel breadth after cooking, maturity, yield plant $^{-1}$, gelatinization temperature and poor grain in parents and cooked kernel length-breadth ratio, all the grades of density of grain except high density grain in hybrids. Heritability and genetic advance were very low for gelatinization temperature in parents which were found to be substantially improved in hybrids with predominant additive gene effect and this could be due to inclusion of specific parents 
involved in hybrid development. Density of grain could be substantially increased towards desirable direction through proportionate reduction in percentage of poor grain constitutions as this character was found to be most responsive to selection towards negative direction and these were predominantly controlled by additive gene effect.

Vanja et al.,(2006) observed additive gene effect on quality characters in rice like kernel length, kernel breadth, kernel length-breadth ratio, amylose content and alkali spreading value though in the present investigation they were found to be controlled by both additive and non-additive gene effect. Similar observation like present finding was given by Veni et al., (2006) in expression of seven physico-chemical quality characters like kernel length, length-breadth ratio, kernel length after cooking, elongation ratio, alkali spreading value and amylose content.

The grain yield and quality of grain in rice are complex characters and their expression are result of cumulative effect of component characters and therefore direct selection for either yield or quality may not produce desirable result and thus an understanding of the relation between the component characters of yield and quality and their relative contribution to dependent character is essential to effect a rational improvement in desirable traits.

Yield is mainly influenced by a number of characters like plant height, effective tillers plant $^{-1}$, panicle length, grain panicle ${ }^{-1}$ and grains plant ${ }^{-1}$. Maturity, amylose content as a main fraction of starch and different grades of rice grains. Gelatinization temperature, a determinant factor of cooked rice is influenced by amylose content and grain structure. The correlation both at phenotypic and genotypic levels between these two dependent variables with their component characters are presented in table 3 and 4 with path analysis in table 5 , 6,7 and 8 .

Genotypic correlation coefficient for all the characters was found to be greater than phenotypic correlation coefficients indicating preponderance of genetic variation in expression of characters. Plant height showed significant positive correlations with grains panicle $^{-1}$, grains plant $^{-1}$, days to $50 \%$ flowering, days to maturity, amylose content in grains and showed significant negative correlation with 1000 grain weight both at genotypic and phenotypic levels. It also showed positive effect though not significant with yield plant ${ }^{-1}$, poor and above average grain density and rest of the characters showed non-significant negative effect with it. Rashid et al., (2002), Zahid et al., (2006) also found like the present investigation significant positive association between plant height and number of grain panicle ${ }^{-1}$.

Effective tillers showed significant and positive correlation with grain yield plant $^{-1}$, grains plant ${ }^{-1}$, grains panicle ${ }^{-1}$, and panicle length. Reddy et al., (1991) also found positive association of panicle length and number of effective tillers with yield plant ${ }^{-1}$.

Grains panicle ${ }^{-1}$ showed significant positive correlation with grains plant ${ }^{-1}$ and these two characters also showed positive association with grain yield, 1000 grain weight, above average grain density and high grain density both at phenotypic and genotypic levels.

Thousand grain weight showed significant positive association with grain yield plant ${ }^{-1}$ both at phenotypic and genotypic levels. Like present investigation Senapati et al., (2008) also showed significant positive association of grain yield with grain plant ${ }^{-1}$ and 1000 -grain weight respectively. Days to $50 \%$ flowering showed significant positive association with maturity and these two characters showed 
non-significant negative association with yield plant $^{-1}$.

Amylose content in grain showed negative correlation with yield plant ${ }^{-1}$ and 1000-grain weight both at phenotypic and genotypic levels.

Percent of high density grain had significant positive association with grain yield both at genotypic and phenotypic levels while percent of above average grain density showed positive but non-signification relation with grain yield at both the levels.

Direct and indirect effect by plant height, number of effective tillers plant ${ }^{-1}, 1000$-grain weight, 50\% flowering, maturity and amylose content on grain yield plant $^{-1}$ were estimated both at phenotypic and genotypic levels. In genotypic path analysis highest positive direct effect was found to be exerted by number of effective tillers plant ${ }^{-1}$ followed by 1000 -grain weight and plant height.

First two characters showed significant correlation with grain yield but plant height showed non-significant positive correlation coefficient with the characters. In phenotypic path analysis highest direct effect was found to be implicated by 1000-grain weight followed by number of effective tillers and plant $^{-1}$ plant height. Veni et al., (2006) observed highest direct effect on yield by test weight. From the findings of present investigation it may be suggested that direct selection through number of effective tillers plant $^{-1}, 1000$-grain weight and plant height may provide scope for yield improvement in rice.

\section{Quality}

Comparatively high positive correlation between amylose content and cooked kernel length and cooked kernel length-breadth ratio was observed both at genotypic and phenotypic levels.

Thousand grain weight showed significant positive association with kernel breadth of uncooked rice and gelatinization temperature while negative correlation with kernel lengthbreadth ratio in uncooked rice and kernel breadth in cooked rice at genotypic level. Kernel length at phenotypic level also showed significant positive correlation with kernel length-breadth ratio of uncooked rice and gelatinization temperature. Kernel breadth showed significant negative association with uncooked kernel length-breadth ratio and gelatinization temperature at genotypic level. Uncooked kernel length-breadth ratio showed significantly positive association with cooked kernel length-breadth ratio and gelatinization temperature at genotypic level. Cooked kernel length at genotypic and phenotypic levels showed positive and significant association with cooked kernel length-breadth ratio and at genotypic level with gelatinization temperature and cooked kernel breadth showed similar correlation but in opposite direction. Zahid (2006) found positive genotypic correlation with 1000-grain weight and gelatinization temperature. Wang et al., (2005) reported negative association between amylose content and gel consistency in indica rice. Roy et al., (2009) failed to observe significant association between amylose content and other quality parameters in rice and they also observed that kernel elongation index was positively associated with kernel length and kernel length-breadth ratio. Zahid et al., (2006) found positive significant correlation with between cooked and uncooked kernel length. Higher direct effect on gelatinization temperature was found to be exerted by cooked kernel length followed by amylose content and thousand grain weight at genotypic level. Uncooked and cooked kernel length breadth ratio also exerted positive direct effect with low in magnitude. 
Table.1 Genetic parameters of different yield and quality parameters (Parents)

\begin{tabular}{|c|c|c|c|c|c|c|c|c|}
\hline \multirow[t]{2}{*}{ Characters } & \multicolumn{3}{|c|}{ Variance } & \multirow[t]{2}{*}{ Heritability } & \multirow[t]{2}{*}{ GCV (\%) } & \multirow[t]{2}{*}{ PCV (\%) } & \multirow[t]{2}{*}{ GA } & \multirow{2}{*}{$\begin{array}{l}\text { GA } \\
(\%) \text { of } \\
\text { mean }\end{array}$} \\
\hline & Environment & Genotypic & Phenotypic & & & & & \\
\hline Plant height (cm) & 0.16 & 499.14 & 499.30 & 1.00 & 22.17 & 22.17 & 46.02 & 45.66 \\
\hline Effective tillers plant ${ }^{-1}$ & 0.30 & 2.45 & 2.75 & 0.89 & 17.05 & 18.07 & 3.04 & 33.16 \\
\hline Panicle length (cm) & 0.58 & 2.44 & 3.02 & 0.81 & 6.89 & 7.67 & 2.89 & 12.76 \\
\hline Grains panicle $^{-1}$ & 5.90 & 181.63 & 187.53 & 0.97 & 10.33 & 10.50 & 27.32 & 20.94 \\
\hline Grains plant $^{-1}$ & 1274.25 & 57044.28 & 58318.53 & 0.98 & 20.55 & 20.78 & 486.60 & 41.87 \\
\hline 1000 grain weight $(\mathrm{g})$ & 0.21 & 2.90 & 3.11 & 0.93 & 8.35 & 8.65 & 3.38 & 16.60 \\
\hline Kernel length (mm) & 0.02 & 0.19 & 0.21 & 0.90 & 6.41 & 6.75 & 0.85 & 12.53 \\
\hline Kernel breadth (mm) & 0.01 & 0.03 & 0.05 & 0.72 & 9.00 & 10.63 & 0.32 & 15.69 \\
\hline Kernel lengthL:Breadth ratio & 0.04 & 0.16 & 0.20 & 0.80 & 11.90 & 13.31 & 0.74 & 21.93 \\
\hline Cooked kernel length (mm) & 0.10 & 0.45 & 0.55 & 0.81 & 8.30 & 9.21 & 1.24 & 15.42 \\
\hline Cooked kernel breadth (mm) & 0.02 & 0.04 & 0.05 & 0.69 & 9.22 & 11.13 & 0.33 & 15.74 \\
\hline $\begin{array}{c}\text { Cooked kernel length:breadth } \\
\text { ratio }\end{array}$ & 0.07 & 0.30 & 0.37 & 0.80 & 13.92 & 15.56 & 1.00 & 25.65 \\
\hline Days to $50 \%$ flowering & 0.26 & 214.37 & 214.63 & 1.00 & 17.75 & 17.76 & 30.14 & 36.54 \\
\hline Days to maturity & 140.16 & 155.73 & 295.89 & 0.53 & 10.85 & 14.95 & 18.65 & 16.21 \\
\hline Yield plant $^{-1}(\mathrm{~g})$ & 5.11 & 10.36 & 15.47 & 0.67 & 15.21 & 18.59 & 5.43 & 25.65 \\
\hline Amylose content $(\%)$ & 0.41 & 8.04 & 8.45 & 0.95 & 13.59 & 13.94 & 5.70 & 27.31 \\
\hline Gelatinization Temperature & 0.78 & 0.02 & 0.79 & 0.02 & 2.94 & 20.07 & 0.04 & 0.89 \\
\hline Poor grain density $(\%)$ & 3.65 & 3.68 & 7.33 & 0.50 & 47.17 & 66.57 & 2.80 & 68.86 \\
\hline Average grain density $(\%)$ & 5.42 & 15.59 & 21.01 & 0.74 & 48.95 & 56.82 & 7.01 & 86.86 \\
\hline Above average grain density $(\%)$ & 11.79 & 58.38 & 70.17 & 0.83 & 51.51 & 56.47 & 14.36 & 96.79 \\
\hline High grain density $(\%)$ & 20.49 & 98.80 & 119.29 & 0.83 & 14.65 & 16.10 & 18.63 & 27.46 \\
\hline
\end{tabular}


Table.2 Genetic parameters of different yield and quality parameters (Hybrids)

\begin{tabular}{|c|c|c|c|c|c|c|c|c|}
\hline \multirow[t]{2}{*}{ Characters } & \multicolumn{3}{|c|}{ Variance } & \multirow[t]{2}{*}{ Heritability } & \multirow[t]{2}{*}{ GCV (\%) } & \multirow[t]{2}{*}{$\operatorname{PCV}(\%)$} & \multirow[t]{2}{*}{ GA } & \multirow{2}{*}{$\begin{array}{c}\text { GA } \\
(\%) \text { of mean }\end{array}$} \\
\hline & environment & Genotypic & Phenotypic & & & & & \\
\hline Plant height (cm) & 0.27 & 364.26 & 364.54 & 1.00 & 20.01 & 20.02 & 39.30 & 41.21 \\
\hline Effective tillers plant ${ }^{-1}$ & 0.22 & 0.76 & 0.98 & 0.78 & 9.22 & 10.45 & 1.59 & 16.77 \\
\hline Panicle length (cm) & 0.10 & 1.95 & 2.05 & 0.95 & 5.37 & 5.51 & 2.80 & 10.78 \\
\hline Grains panicle $^{-1}$ & 1.57 & 113.67 & 115.24 & 0.99 & 7.48 & 7.53 & 21.81 & 15.31 \\
\hline Grains plant $^{-1}$ & 1742.01 & 15840.40 & 17582.41 & 0.90 & 10.02 & 10.55 & 246.09 & 19.58 \\
\hline 1000 grain weight (g) & 5.69 & 0.76 & 6.44 & 0.12 & 4.07 & 11.88 & 0.61 & 2.88 \\
\hline Kernel length (mm) & 0.01 & 0.14 & 0.14 & 0.95 & 5.08 & 5.21 & 0.74 & 10.20 \\
\hline Kernel breadth (mm) & 0.00 & 0.01 & 0.01 & 0.80 & 3.76 & 4.20 & 0.14 & 6.93 \\
\hline Kernel length:Breadth ratio & 0.01 & 0.04 & 0.05 & 0.82 & 5.56 & 6.12 & 0.37 & 10.40 \\
\hline Cooked kernel length (mm) & 0.07 & 0.48 & 0.55 & 0.87 & 6.94 & 7.44 & 1.33 & 13.35 \\
\hline Cooked kernel breadth (mm) & 0.04 & 0.13 & 0.17 & 0.76 & 13.40 & 15.37 & 0.64 & 24.07 \\
\hline Cooked kernel length:breadth ratio & 0.10 & 0.15 & 0.25 & 0.59 & 10.09 & 13.16 & 0.60 & 15.94 \\
\hline Days to $50 \%$ flowering & 0.23 & 41.21 & 41.44 & 0.99 & 8.72 & 8.74 & 13.19 & 17.91 \\
\hline Days to maturity & 0.34 & 131.36 & 131.70 & 1.00 & 10.58 & 10.59 & 23.58 & 21.77 \\
\hline Yield plant $^{-1}(\mathrm{~g})$ & 0.22 & 10.14 & 10.35 & 0.98 & 13.05 & 13.18 & 6.49 & 26.59 \\
\hline Amylose content $(\%)$ & 0.37 & 2.56 & 2.93 & 0.87 & 7.12 & 7.62 & 3.08 & 13.70 \\
\hline Gelatinization Temperature & 0.14 & 1.73 & 1.87 & 0.93 & 27.23 & 28.28 & 2.61 & 54.01 \\
\hline Poor grain density $(\%)$ & 3.68 & 1.40 & 5.08 & 0.28 & 33.42 & 63.62 & 1.28 & 36.17 \\
\hline Average grain density $(\%)$ & 8.39 & 6.57 & 14.96 & 0.44 & 42.42 & 64.02 & 3.50 & 57.90 \\
\hline Above average grain density $(\%)$ & 20.11 & 14.26 & 34.37 & 0.41 & 20.37 & 31.62 & 5.01 & 27.02 \\
\hline High grain density $(\%)$ & 27.09 & 81.11 & 108.20 & 0.75 & 13.30 & 15.36 & 16.06 & 23.72 \\
\hline
\end{tabular}


Table.3 Genotypic and phenotypic correlation for yield and some yield attributing characters

\begin{tabular}{|c|c|c|c|c|c|c|c|c|c|c|c|c|c|}
\hline & ET & PL & GP & GPL & GW & $50 \%$ & MT & $\mathbf{A C}$ & Poor & Avg & GD & HD & $\mathbf{Y P}$ \\
\hline $\begin{array}{c}\text { PH (G) } \\
\quad(P)\end{array}$ & $\begin{array}{l}-0.10 \\
-0.09\end{array}$ & $\begin{array}{c}0.50 * * \\
0.41 *\end{array}$ & $\begin{array}{l}0.62 * * \\
0.53 * *\end{array}$ & $\begin{array}{c}0.58 * * \\
0.46^{*}\end{array}$ & $\begin{array}{r}-0.55 * * \\
0.50 * *\end{array}$ & $\begin{array}{l}0.67 * * \\
0.63 * *\end{array}$ & $\begin{array}{l}0.63 * * \\
0.58 * *\end{array}$ & $\begin{array}{l}0.43^{*} \\
0.41^{*}\end{array}$ & $\begin{array}{l}0.20 \\
0.15\end{array}$ & $\begin{array}{l}-0.09 \\
-0.08\end{array}$ & $\begin{array}{l}0.05 \\
0.03\end{array}$ & $\begin{array}{l}-0.12 \\
-0.11\end{array}$ & $\begin{array}{l}0.15 \\
0.09\end{array}$ \\
\hline $\begin{array}{c}\text { ET (G) } \\
\quad(\mathbf{P})\end{array}$ & & $\begin{array}{l}0.81 * * \\
0.72 * *\end{array}$ & $\begin{array}{l}0.67 * * \\
0.65 * *\end{array}$ & $\begin{array}{l}0.80 * * \\
0.74 * *\end{array}$ & $\begin{array}{l}-0.35 \\
-0.32\end{array}$ & $\begin{array}{l}0.03 \\
0.03\end{array}$ & $\begin{array}{l}-0.05 \\
-0.01\end{array}$ & $\begin{array}{l}0.26 \\
0.24\end{array}$ & $\begin{array}{l}-0.17 \\
-0.11\end{array}$ & $\begin{array}{c}0.42 * \\
0.34\end{array}$ & $\begin{array}{l}0.34 \\
0.31\end{array}$ & $\begin{array}{l}-0.37 \\
-0.31\end{array}$ & $\begin{array}{c}0.55 * * \\
0.49 *\end{array}$ \\
\hline $\begin{array}{c}\text { PL (G) } \\
\quad(\mathbf{P})\end{array}$ & & & $\begin{array}{l}0.39 \\
0.32\end{array}$ & $\begin{array}{c}0.41 * \\
0.35\end{array}$ & $\begin{array}{l}0.20 \\
0.15\end{array}$ & $\begin{array}{l}0.48 * \\
0.45 *\end{array}$ & $\begin{array}{l}0.34 \\
0.28\end{array}$ & $\begin{array}{l}0.11 \\
0.09\end{array}$ & $\begin{array}{l}-0.38 \\
-0.28\end{array}$ & $\begin{array}{l}-0.11 \\
-0.09\end{array}$ & $\begin{array}{l}0.39 \\
0.35\end{array}$ & $\begin{array}{l}0.31 \\
0.28\end{array}$ & $\begin{array}{l}0.37 \\
0.32\end{array}$ \\
\hline $\begin{array}{c}\text { GP }(\mathbf{G}) \\
\quad(\mathbf{P})\end{array}$ & & & & $\begin{array}{l}0.57 * * \\
0.55 * *\end{array}$ & $\begin{array}{l}0.34 \\
0.31\end{array}$ & $\begin{array}{l}-0.20 \\
-0.19\end{array}$ & $\begin{array}{l}-0.14 \\
-0.11\end{array}$ & $\begin{array}{l}0.24 \\
0.23\end{array}$ & $\begin{array}{l}-0.23 \\
-0.20\end{array}$ & $\begin{array}{l}0.19 \\
0.16\end{array}$ & $\begin{array}{l}0.39 \\
0.34\end{array}$ & $\begin{array}{l}0.35 \\
0.31\end{array}$ & $\begin{array}{l}0.35 \\
0.31\end{array}$ \\
\hline $\begin{array}{c}\text { GPL (G) } \\
(\mathbf{P})\end{array}$ & & & & & $\begin{array}{l}0.15 \\
0.14\end{array}$ & $\begin{array}{l}-0.05 \\
-0.05\end{array}$ & $\begin{array}{l}-0.11 \\
-0.09\end{array}$ & $\begin{array}{l}0.03 \\
0.02\end{array}$ & $\begin{array}{l}-0.20 \\
-0.15\end{array}$ & $\begin{array}{c}0.46^{*} \\
0.39\end{array}$ & $\begin{array}{l}0.31 \\
0.21\end{array}$ & $\begin{array}{l}0.25 \\
0.21\end{array}$ & $\begin{array}{l}0.37 \\
0.34\end{array}$ \\
\hline $\begin{array}{c}\text { GW (G) } \\
(\mathrm{P})\end{array}$ & & & & & & $\begin{array}{l}-0.42 * \\
-0.40 *\end{array}$ & $\begin{array}{l}-0.37 \\
-0.32\end{array}$ & $\begin{array}{l}-0.13 \\
-0.12\end{array}$ & $\begin{array}{l}-0.34 \\
-0.21\end{array}$ & $\begin{array}{l}0.39 \\
0.34\end{array}$ & $\begin{array}{l}0.25 \\
0.21\end{array}$ & $\begin{array}{l}0.35 \\
0.29\end{array}$ & $\begin{array}{l}0.49 * \\
0.42 *\end{array}$ \\
\hline $\begin{array}{c}50 \%(G) \\
(P)\end{array}$ & & & & & & & $\begin{array}{l}0.83 * * \\
0.69 * *\end{array}$ & $\begin{array}{l}-0.24 \\
-0.24\end{array}$ & $\begin{array}{l}0.11 \\
0.07\end{array}$ & $\begin{array}{l}0.15 \\
0.13\end{array}$ & $\begin{array}{l}-0.03 \\
-0.02\end{array}$ & $\begin{array}{c}-0.08 \\
0.07\end{array}$ & $\begin{array}{l}-0.26 \\
-0.22\end{array}$ \\
\hline $\begin{array}{c}\text { MT (G) } \\
\quad(\mathbf{P})\end{array}$ & & & & & & & & $\begin{array}{l}-0.39 \\
-0.31\end{array}$ & $\begin{array}{l}0.18 \\
0.15\end{array}$ & $\begin{array}{l}0.27 \\
0.19\end{array}$ & $\begin{array}{l}0.18 \\
0.15\end{array}$ & $\begin{array}{l}-0.28 \\
-0.19\end{array}$ & $\begin{array}{l}-0.23 \\
-0.15\end{array}$ \\
\hline $\begin{array}{c}\mathrm{AC}(\mathrm{G}) \\
\quad(\mathrm{P})\end{array}$ & & & & & & & & & $\begin{array}{l}-0.01 \\
-0.01\end{array}$ & $\begin{array}{l}0.01 \\
0.01\end{array}$ & $\begin{array}{l}-0.21 \\
-0.17\end{array}$ & $\begin{array}{l}0.21 \\
0.18\end{array}$ & $\begin{array}{l}-0.28 \\
-0.21\end{array}$ \\
\hline $\begin{array}{c}\text { Poor (G) } \\
\text { (P) }\end{array}$ & & & & & & & & & & $\begin{array}{c}0.54 * * \\
0.37\end{array}$ & $\begin{array}{l}-0.15 \\
-0.17\end{array}$ & $\begin{array}{l}-0.45 * \\
-0.47 *\end{array}$ & $\begin{array}{l}0.09 \\
0.08\end{array}$ \\
\hline $\begin{array}{c}\operatorname{AVG}(\mathbf{G}) \\
(\mathbf{P})\end{array}$ & & & & & & & & & & & $\begin{array}{l}-0.05 \\
-0.09\end{array}$ & $\begin{array}{l}-0.55^{*} * \\
-0.57 * *\end{array}$ & $\begin{array}{l}0.26 \\
0.21\end{array}$ \\
\hline $\begin{array}{c}\mathbf{A A V G}(\mathbf{G}) \\
(P)\end{array}$ & & & & & & & & & & & & $\begin{array}{l}-0.72 * * \\
-0.75 * *\end{array}$ & $\begin{array}{l}0.38 \\
0.35 \\
\end{array}$ \\
\hline $\begin{array}{c}\text { HD (G) } \\
\text { (P) }\end{array}$ & & & & & & & & & & & & & $\begin{array}{c}0.46^{*} \\
0.39\end{array}$ \\
\hline
\end{tabular}

$*$,**=significant at 5\%, $1 \%$ level. $[\mathrm{PH}=$ plant height, ET= effective tillers per plant, $\mathrm{PL}=$ panicle length, GP $=$ no. of grains per panicle, GPL= no. of grains per plant, $\mathrm{GW}=1000$ grain weight, $50 \%$ = days to $50 \%$ flowering, $\mathrm{MT}=$ Days to maturity, $\mathrm{AC}=$ Amylose Content, Poor $=$ percentage of poor seed, $\mathrm{AVG}=$

percentage of average seed density, $\mathrm{AAVG}=$ Percentage of above average seed density, $\mathrm{HD}=$ high seed density percentage $(\mathrm{G})=\mathrm{Genotypic},(\mathrm{P})=\mathrm{Phenotypic}$. 
Table.4 Genotypic and Phenotypic correlation for some quality parameters

\begin{tabular}{|c|c|c|c|c|c|c|c|c|}
\hline & $\begin{array}{l}\text { 1000- } \\
\text { Grain } \\
\text { weight } \\
\text { (g) }\end{array}$ & $\begin{array}{l}\text { Kernel } \\
\text { length } \\
(\mathrm{mm})\end{array}$ & $\begin{array}{l}\text { Kernel breadth } \\
(\mathbf{m m})\end{array}$ & K L:B ratio & CKL (mm) & $\begin{array}{l}\text { CKB } \\
(\mathbf{m m})\end{array}$ & $\begin{array}{c}\text { CKL:B } \\
\text { ratio }\end{array}$ & $\begin{array}{c}\text { Gelatinization } \\
\text { Temperature }\end{array}$ \\
\hline $\begin{array}{c}\text { Amylose (G) } \\
\text { content }(\%)(P)\end{array}$ & $\begin{array}{l}-0.13 \\
-0.13\end{array}$ & $\begin{array}{l}-0.10 \\
-0.10\end{array}$ & $\begin{array}{l}-0.20 \\
-0.15\end{array}$ & $\begin{array}{l}0.07 \\
0.05\end{array}$ & $\begin{array}{l}0.31 \\
0.26\end{array}$ & $\begin{array}{l}-0.20 \\
-0.14\end{array}$ & $\begin{array}{l}0.30 \\
0.24\end{array}$ & $\begin{array}{l}0.08 \\
0.00\end{array}$ \\
\hline $\begin{array}{c}\text { Kernel (G) } \\
\text { length }(\mathbf{m m})(\mathbf{P})\end{array}$ & & & $\begin{array}{l}-0.13 \\
-0.12 \\
\end{array}$ & $\begin{array}{l}0.67 * * \\
0.63 * *\end{array}$ & $\begin{array}{l}0.23 \\
0.16 \\
\end{array}$ & $\begin{array}{l}-0.14 \\
-0.13\end{array}$ & $\begin{array}{l}0.25 \\
0.20 \\
\end{array}$ & $\begin{array}{c}0.42 * \\
0.01\end{array}$ \\
\hline $\begin{array}{c}\text { Kernel }(\mathbf{G}) \\
\text { breadth }(\mathbf{m m}) \\
(\mathbf{P})\end{array}$ & & & & $\begin{array}{l}-0.82 * * \\
-0.84 * *\end{array}$ & $\begin{array}{l}-0.28 \\
-0.20\end{array}$ & $\begin{array}{l}1.00 * * \\
0.99 * *\end{array}$ & $\begin{array}{l}-0.81 * * \\
-0.80 * *\end{array}$ & $\begin{array}{l}-0.47 * \\
-0.02\end{array}$ \\
\hline $\begin{array}{c}\text { K L:B ratio (G) } \\
(\mathbf{P})\end{array}$ & & & & & $\begin{array}{l}0.33 \\
0.24\end{array}$ & $\begin{array}{l}-0.83 * * \\
-0.84 * *\end{array}$ & $\begin{array}{l}0.75 * * \\
0.73 * *\end{array}$ & $\begin{array}{c}0.51 * * \\
0.01\end{array}$ \\
\hline $\begin{array}{c}\mathrm{CKL}(\mathbf{m m})(\mathbf{G}) \\
(\mathbf{P})\end{array}$ & & & & & & $\begin{array}{l}-0.28 \\
-0.20\end{array}$ & $\begin{array}{l}0.78 * * \\
0.74 * *\end{array}$ & $\begin{array}{c}0.85^{* *} \\
0.10\end{array}$ \\
\hline $\begin{array}{l}\text { CKB (G) } \\
(\mathbf{m m})(\mathbf{P})\end{array}$ & & & & & & & $\begin{array}{l}-0.82 * * \\
-0.80 * *\end{array}$ & $\begin{array}{c}-0.81 * * \\
-0.02\end{array}$ \\
\hline $\begin{array}{c}\text { CKL:B ratio } \\
\text { (G) } \\
\text { (P) }\end{array}$ & & & & & & & & $\begin{array}{c}0.75^{* *} * \\
0.06\end{array}$ \\
\hline
\end{tabular}

$*$,**=significant at $5 \%, 1 \%$ level.

$[\mathrm{CKL}=$ cooked kernel length, $\mathrm{CKB}=$ cooked kernel breadth, $(\mathrm{G})=$ Genotypic, $(\mathrm{P})=$ Phenotypic $]$. 
Table.5 Genotypic path analysis for yield attributing characters on yield

\begin{tabular}{|c|c|c|c|c|c|c|c|c|c|c|}
\hline $\begin{array}{l}\text { Sl. } \\
\text { No. }\end{array}$ & Characters & $\begin{array}{c}\text { Plant } \\
\text { height } \\
(\mathrm{cm})\end{array}$ & $\begin{array}{c}\text { Effective } \\
\text { tillers } \\
\text { Plant }^{-1}\end{array}$ & $\begin{array}{c}\text { Panicle } \\
\text { length } \\
\text { (cm) }\end{array}$ & $\begin{array}{c}\text { Grains } \\
\text { panicle }^{-1}\end{array}$ & $\begin{array}{l}\text { Grains } \\
\text { plant }^{-1}\end{array}$ & $\begin{array}{l}\text { 1000- } \\
\text { grain } \\
\text { wt. }\end{array}$ & $\begin{array}{c}\text { Days to } \\
\mathbf{5 0 \%} \\
\text { flowering }\end{array}$ & $\begin{array}{c}\text { Days to } \\
\text { Maturity }\end{array}$ & $\begin{array}{c}\text { Yield } \\
\text { plant }^{-1} \\
(\%)\end{array}$ \\
\hline 1 & Plant height $(\mathrm{cm})$ & 0.374 & -0.068 & 0.062 & -0.036 & 0.148 & -0.232 & -0.076 & -0.204 & 0.15 \\
\hline 3 & Panicle length $(\mathrm{cm})$ & 0.122 & 0.195 & 0.083 & 0.094 & -0.201 & 0.114 & -0.048 & -0.110 & 0.37 \\
\hline 4 & Grains panicle $^{-1}$ & 0.046 & 0.346 & 0.001 & 0.198 & -0.384 & 0.143 & 0.022 & 0.039 & 0.35 \\
\hline 5 & Grains plant $^{-1}$ & -0.033 & 0.568 & 0.403 & 0.114 & -0.669 & -0.061 & 0.005 & 0.036 & 0.37 \\
\hline 7 & Days to $50 \%$ flowering & 0.170 & 0.023 & 0.004 & -0.039 & 0.031 & -0.174 & -0.114 & -0.296 & -0.26 \\
\hline 8 & Days to Maturity & 0.159 & -0.036 & 0.003 & -0.023 & 0.075 & -0.154 & 0.094 & -0.326 & -0.23 \\
\hline
\end{tabular}

Table.6 Phenotypic path analysis for yield attributing characters on yield

\begin{tabular}{|c|c|c|c|c|c|c|c|c|c|c|}
\hline $\begin{array}{l}\text { Sl. } \\
\text { No. }\end{array}$ & Characters & $\begin{array}{c}\text { Plant } \\
\text { height } \\
\text { (cm) }\end{array}$ & $\begin{array}{c}\text { Effectiv } \\
\text { e tiller } \\
\text { plant }^{-1}\end{array}$ & $\begin{array}{c}\text { Panicle } \\
\text { length } \\
\text { (cm) }\end{array}$ & $\begin{array}{c}\text { Grains } \\
\text { panicle }^{-1}\end{array}$ & $\begin{array}{l}\text { Grains } \\
\text { plant- }\end{array}$ & $\begin{array}{c}1000 \\
\text { grain } \\
\text { weight }\end{array}$ & $\begin{array}{c}\text { Days to } \\
50 \% \\
\text { flowering }\end{array}$ & $\begin{array}{l}\text { Days to } \\
\text { maturity }\end{array}$ & $\begin{array}{c}\text { Yield } \\
\text { plant }^{-1}\end{array}$ \\
\hline 1 & Plant height $(\mathrm{cm})$ & 0.325 & -0.028 & -0.035 & 0.056 & -0.021 & -0.280 & -0.137 & 0.146 & 0.09 \\
\hline 3 & Panicle length $(\mathrm{cm})$ & 0.133 & 0.087 & -0.202 & -0.010 & 0.119 & 0.104 & -0.077 & 0.078 & 0.32 \\
\hline 4 & Grains panicle $^{-1}$ & 0.059 & 0.462 & -0.014 & -0.140 & 0.129 & 0.205 & 0.079 & -0.001 & 0.31 \\
\hline 6 & 1000 -grain wt. & -0.1730 & -0.031 & -0.010 & -0.044 & -0.023 & 0.526 & 0.082 & -0.003 & 0.42 \\
\hline 7 & $\begin{array}{l}\text { Days to } 50 \% \\
\text { flowering }\end{array}$ & 0.217 & 0.003 & -0.076 & 0.027 & -0.007 & -0.210 & -0.205 & 0.009 & -0.22 \\
\hline 8 & Days to Maturity & 0.147 & -0.071 & -0.029 & 0.082 & -0.014 & -0.26483 & -0.124 & 0.015 & -0.15 \\
\hline
\end{tabular}


Table.7 Genotypic path analysis for quality attributing characters on gelatinization temperature

\begin{tabular}{|c|c|c|c|c|c|c|c|c|c|}
\hline $\begin{array}{l}\text { Sl. } \\
\text { No }\end{array}$ & Characters & $\begin{array}{c}\text { Amylose } \\
\text { Content }\end{array}$ & $\begin{array}{c}\text { Kernel } \\
\text { length } \\
(\mathbf{m m})\end{array}$ & $\begin{array}{c}\text { Kernel } \\
\text { breadth } \\
(\mathbf{m m})\end{array}$ & $\begin{array}{c}\text { K L:B } \\
\text { Ratio }\end{array}$ & $\begin{array}{l}\text { Cooked } \\
\text { kernel } \\
\text { length } \\
(\mathbf{m m})\end{array}$ & $\begin{array}{c}\text { Cooked } \\
\text { kernel } \\
\text { breadth } \\
(\mathbf{m m})\end{array}$ & $\begin{array}{l}\text { CKL: } \\
\text { B ratio }\end{array}$ & $\begin{array}{c}\text { Gelatinizatio } \\
\text { n } \\
\text { Temperature }\end{array}$ \\
\hline 1 & $\begin{array}{c}\text { Amylose } \\
\text { content }(\%)\end{array}$ & 17.413 & 1.592 & 1.661 & 1.017 & -20.536 & -0.096 & 0.235 & 0.08 \\
\hline 2 & $\begin{array}{c}\text { Kernel } \\
\text { length }(\mathrm{mm})\end{array}$ & -6.530 & -4.245 & -1.331 & 1.676 & 13.109 & -3.540 & 0.087 & 0.42 \\
\hline 3 & $\begin{array}{l}\text { Kernel breadth } \\
(\mathrm{mm})\end{array}$ & -4.214 & -0.823 & -6.862 & 1.555 & 10.572 & -3.660 & 0.208 & -0.47 \\
\hline 4 & K L:B ratio & 3.221 & -1.295 & -1.942 & 5.496 & -8.220 & -0.060 & -0.038 & 0.51 \\
\hline 5 & $\begin{array}{l}\text { Cooked kernel } \\
\text { length }(\mathrm{mm})\end{array}$ & -13.530 & -2.105 & -2.745 & -1.709 & 26.430 & -6.249 & 0.203 & 0.85 \\
\hline 6 & $\begin{array}{c}\text { Cooked kernel } \\
\text { breadth }(\mathrm{mm})\end{array}$ & 0.139 & -1.248 & -2.086 & 0.027 & 13.717 & -12.041 & 0.770 & -0.81 \\
\hline 7 & C KL:B Ratio & 4.318 & -0.390 & -1.510 & -0.220 & 5.656 & -9.789 & 0.946 & 0.75 \\
\hline
\end{tabular}

Residual effect $=0.333923$

Table.8 Phenotypic path analysis for quality attributing characters on gelatinization temperature

\begin{tabular}{|c|c|c|c|c|c|c|c|c|c|}
\hline $\begin{array}{l}\text { Sl. } \\
\text { No }\end{array}$ & Characters & $\begin{array}{c}\text { Amylose } \\
\text { content } \\
(\%)\end{array}$ & $\begin{array}{c}\text { Kernel } \\
\text { length } \\
(\mathbf{m m})\end{array}$ & $\begin{array}{c}\text { Kernel } \\
\text { breadth } \\
(\mathbf{m m})\end{array}$ & $\begin{array}{c}\text { K L:B } \\
\text { ratio }\end{array}$ & $\begin{array}{c}\text { Cooked } \\
\text { kernel } \\
\text { length } \\
(\mathrm{mm})\end{array}$ & $\begin{array}{c}\text { Cooked } \\
\text { kernel } \\
\text { breadth } \\
(\mathbf{m m})\end{array}$ & $\begin{array}{l}\text { C Kl:B } \\
\text { Ratio }\end{array}$ & $\begin{array}{c}\text { Gelatinization } \\
\text { Temperature }\end{array}$ \\
\hline 1 & $\begin{array}{l}\text { Amylose } \\
\text { content (\%) }\end{array}$ & -0.386 & -0.042 & -0.140 & 0.004 & -0.020 & -0.001 & 0.072 & 0.00 \\
\hline 3 & $\begin{array}{c}\text { Kernel } \\
\text { length }(\mathrm{mm})\end{array}$ & 0.141 & 0.116 & 0.109 & 0.007 & 0.012 & -0.058 & 0.025 & 001 \\
\hline 4 & $\begin{array}{l}\text { Kernel breadth } \\
(\mathrm{mm})\end{array}$ & 0.093 & 0.022 & 0.580 & 0.007 & 0.007 & -0.061 & 0.064 & -0.02 \\
\hline 5 & K L:B ratio & -0.068 & 0.031 & 0.152 & $\mathbf{0 . 0 2 7}$ & -0.007 & 0.000 & -0.011 & 0.01 \\
\hline 6 & $\begin{array}{l}\text { Cooked kernel } \\
\text { length }(\mathrm{mm})\end{array}$ & 0.102 & 0.018 & 0.056 & -0.002 & 0.076 & -0.034 & 0.017 & 0.10 \\
\hline 7 & $\begin{array}{l}\text { Cooked kernel } \\
\text { breadth }(\mathrm{mm})\end{array}$ & -0.003 & 0.033 & 0.176 & -0.000 & 0.012 & -0.204 & 0.239 & -0.02 \\
\hline 8 & C Kl:B Ratio & -0.095 & 0.010 & 0.127 & -0.001 & 0.004 & -0.165 & 0.294 & 0.06 \\
\hline
\end{tabular}

Residual effect $=0.4024853$

Kernel length as well as length-breadth ratio of cooked and uncooked rice showed significant positive correlation with gelatinization temperature. Kernel breadth showed significant negative association with gelatinization temperature both at genotypic and phenotypic levels. Roy et al., (2009) observed that kernel elongation index was positively associated with kernel length and kernel length-breadth ratio. Zahid et al., 
(2006) found positive significant correlation between cooked and uncooked kernel length. For improved cooking fine rice grains are preferred as kernel length had shown positive association with gelatinization temperature. The characters like amylose content, 1000grain weight, kernel length, breadth and their ratio before and after cooking were considered to analyse direct and indirect effect of these parameters on gelatinization temperature. Thousand grain weight, kernel length and length-breadth ratio before and after cooking showed significant association with gelatinization temperature but kernel breadth before and after cooking showed negative association with it and amylose content showed numerically very low correlationcoefficient. At genotypic level highest direct effect on gelatinization temperature was exerted by cooked kernel length, 1000-grain weight and cooked kernel length- breadth ratio.

\section{References}

FAO., 2007. Report of the $33^{\text {rd }}$ session of the committee on food security. Rome, Italy.

Kempthrone, O., 1957. An introduction to genetic studies. John Wiley and Sons, New York, USA.

Roy, S., Banerjee, A., Senapati, B.K. 2009. Evaluation of some aromatic rice germplasm in new alluviul soil of West Bengal. Environment and Ecolog. 27(3A): 1240-1242.

Rashid, M.S., H.A. Sadaqat and M, Babar 2002. Correlation and path co-efficient analysis for yield and its components in rice. Asian J. Pl. Sci., 1(3): 241-244.

Reddy, CDR., Nerker, YS., and Dhanalaxmi, G., 1991. Heterosis and inbreeding depression for grain size and yield in rice (Oryza sativa L.) under direct seeding. Indian J. of Agricultural Sciences. 61(6): 416-419.

Senapati, B.K., Mandal, G.S., Pal, S., Sarkar, G., and Sarkar, K.K., 2008. Evaluation of some high yielding genotypes of rice under new alluvial zone of West Bengal. Environment and Ecology. 26(3): 1010-1015.

Tomar, J.B., and J.S. Nanda., 1982. Correlation between quality traits in rice. Oryza. 19: 13-18.

Vanja,T., and Luckins, C. Babu. 2006. Variability in grain quality attributers of high yielding rice varieties (Oryza sativa L.) of diverse origin. Journal of Tropical Agriculture. 44(1-2): 61-63.

Veni, B.K., and Rani, N.S., 2006. Association of grain yield with quality characteristics and other yield components in rice. Oryza. 43:320322.

Wang, D.Y., Zhang, X.F., Zhu, Z.W., Chen, N., Min, J., Yao, Q., Yan, J., Liao, X.Y., 2005. Correlation analysis of rice grain quality characteristics. Acta Agronomica. 31(8): 1086-1091.

Zahid, M.A., M, Akhtar., M, Sabir., Z, Manzoor., and T, Awan., 2006. Correlation and path analysis studies of yield and economic traits in Basmati rice (Oryza sativa L.). Asian Pl. Sci. 5(4): 643-64.

\section{How to cite this article:}

Debashis Saren, Subhra Mukherjee and Rajib Tudu. 2019. Genetic Variability and Correlation Study for Yield and Quality Characters in Rice. Int.J.Curr.Microbiol.App.Sci. 8(12): 21432153. doi: https://doi.org/10.20546/ijcmas.2019.812.254 